\title{
Efficacy of Acupuncture for Persistent and Intractable Hiccups: A Protocol for Systematic Review and Meta Analysis of Randomized Controlled Trials
}

\section{Yu Zhang}

The First Affiliated Hospital of Anhui University of Chinese Medicine

\section{Xudong Jiang}

The First Affiliated Hospital of Anhui University of Chinese Medicine

\section{Zhijie Wang}

Shanxi Province Hospital of Traditional Chinese Medical

\section{Mingming $\mathrm{He}$}

Shaaxi Traditional Chinese Medicine Hospital

\section{Zimeng Lv}

The First Affiliated Hospital of Anhui University of Chinese Medicine

\section{Gaiqin Yang}

Shaaxi Traditional Chinese Medicine Hospital

Weixun Qin ( $\nabla$ qinweixun@foxmail.com )

Shaanxi Traditonal Chinese Medicine Hospital https://orcid.org/0000-0001-8512-5686

\section{Protocol}

Keywords: persistent and intractable hiccups, meta analysis, acupuncture, protocol, systematic review

Posted Date: October 12th, 2020

DOI: https://doi.org/10.21203/rs.3.rs-88175/v1

License: (9) (i) This work is licensed under a Creative Commons Attribution 4.0 International License. Read Full License

Version of Record: A version of this preprint was published at Medicine on February 26th, 2021. See the published version at https://doi.org/10.1097/MD.0000000000024879. 


\section{Abstract}

Background: Persistent and intractable hiccups are a common clinical symptom that cause considerable physical pain to patients and severely damage their quality of lives. An increasing number of studies have demonstrated that acupuncture applied at acupoints dominated by Cuanzhu (BL2) can be used as one of the nonpharmacological therapies for controlling intractable hiccups. However, there is insufficient evidence evaluating the safety and effectiveness of those interventions. Therefore, this study is intended to conduct a systematic review and meta-analysis to provide evidence for a further study investigating alternative treatment options for persistent and intractable hiccups.

Methods: Randomized controlled trials (RCTs) of adult patients aged $>18$ years who meet the criteria for intractable hiccup diagnosis will be included, regardless of gender, nationality, and education level. Eight electronic databases will be searched, including four Chinese databases (CNKI, SinoMed, Wanfang Database, and Chinese Scientific Journal Database), four English databases (Web of Science, Medline, Embase, and Cochrane Library), from their date of establishment to September 2020. Two independent reviewers will evaluate the title summary for each RCT. Disagreements will be discussed with a third commentator. Data integration, heterogeneity analysis, subgroup analysis, and sensitivity analysis, will be performed using R-3.3.2 software. The RevMan 5.3 software will be used for the meta-analysis, and the "risk of bias" assessment will be conducted based on the methodological quality of the included trials recommended by the Cochrane Handbook 5.1. The quality evaluation of this study will be completed by the Grading of Recommendations, Assessment, Development, and Evaluation.

Discusstion: This systematic review will provide evidence to assess the validity and safety of applying acupuncture at acupoints dominated by Cuanzhu (BL2) for persistent and intractable hiccups, which may provide clinicians with more choices in the treatment of this disease.

\section{Systematic review registration: PROSPERO CRD42020114900}

\section{Background}

\subsection{Description of the condition}

Hiccups are caused by the stimulation of the phrenic nerve and the vagus nerve, and the diaphragm and intercostal muscles are involuntarily synchronized with strong rhythmic contraction, causing the throat to produce a short, loud sound. A retrospective study reported that 84 patients were diagnosed with hiccups in Kirikkale University Medical Faculty Emergency Department from 2013-2018. Duration of hiccups attack was $<48 \mathrm{~h}$ in $44.1 \%$ of patients, between $48 \mathrm{~h}$ and 1 month (persistent) in $36.9 \%$, and longer than 1 month (intractable) in 19\%.[1] Based on duration, hiccups are classified as intractable that last for more than 30 days.[2] The incidence of intractable hiccups is relatively high when combined with certain underlying disorders such as Parkinson's disease, stroke, advanced tumors, and gastroesophageal reflux disease.[3-5] Studies have reported that approximately $10 \%$ of patients with gastroesophageal reflux disease and $20 \%$ of patients with Parkinson's disease may develop a hiccup at the time of consultation, 
whereas only $3 \%$ of individuals without these conditions could develop a hiccup.[6, 7] In addition to causing severe discomfort and pain, intractable hiccups can cause attention deficit, malnutrition, and weight loss, leading to fatigue, insomnia, anxiety, depression, and reduced quality of life of the patient.[8, 9]

Clinically, a variety of different medical drugs are used to treat intractable hiccups, such as diazepam, quinine, chlorpromazine, metoclopramide, and baclofen;[10] however, the evidence related to the use of these drugs has been primarily derived from case reports, which necessitates further studies to confirm their effectiveness. In cases where drug treatments are ineffective, it has been reported that surgical methods can be used to relieve the compression.[11] Moreover, alternative treatment options such as hypnosis, breath holding, and swallowing dry granulated sugar have also been proposed to control intractable hiccups.[12] However, drugs and surgical treatments may result in varying degrees of adverse reactions, and alternative therapies lack high-quality evidence to support their efficacy.

Acupoints dominated by Cuanzhu (BL2) for controlling persistent and intractable hiccups has been widely used in alternative treatment methods and confirmed to be more effective than other therapies.[1319] However, there is no systematic review and meta-analysis to evaluate the potential benefits and harms of applying acupuncture at acupoints dominated by Cuanzhu (BL2) in the treatment of intractable hiccups. Therefore, in case this treatment proves to be effective, we could obtain a rapid, safe, and effective method to help patients relieve their conditions.

\subsection{Description of the intervention}

Numerous clinical trials have demonstrated that acupuncture can treat intractable hiccups, including acupressure, acupoint injection, electroacupuncture, and acupoint embedding.[13-19] Previous studies have also suggested that acupuncture is effective as an adjunct replacement therapy for intractable hiccups after stroke.[20] A systematic review of Cochrane included four RCTs evaluating the effectiveness of different acupuncture treatments for intractable hiccups.[21] Although all four studies suggested that acupuncture is effective in the treatment of intractable hiccups, it is unfortunate that none of the four studies provided a placebo control group, nor did they explain the side effects and complications.

Cuanzhu (BL2) is an acupoint located on the medial end of the eyebrow (Figure 1). We selected Cuanzhu as it belongs to the Bladder Meridian of Foot-Taiyang and has the effect of lowering the adverse qi (a disorder of qi leads to hiccups according to the traditional Chinese medicine theory). It is the empirical point in Chinese medicine acupuncture treatment for hiccups. Studies have reported that the total effective rates were $96.4 \%$ in the treatment group (using both thumbs to press the two Cuanzhu to treat the hiccups, with the power ranging from light to heavy for 2-3 min and the patient taking a deep breath and holding their breath for $>30 \mathrm{~s}$ ) and $81.5 \%$ in the control group (using baclofen). [22] Another study reported that based on conventional treatment, the effective rate of acupuncture on both Cuanzhu acupoints of patients with secondary intractable hiccups after traumatic brain injury, was $93.18 \%$, which was significantly better than that (69.05\%) in the control group (using chlorpromazine).[23] Although 
acupressure or acupuncture combined with other techniques has been widely used to treat intractable hiccups, there are no standardized treatments in the clinic. In addition, there is a lack of more objectivecontrolled studies comparing the various methods, due to which we cannot judge the difference in efficacy between the various methods.

\section{Objective}

The purpose of this systematic review is to conduct a meta-analysis to evaluate the efficacy and safety of acupuncture at acupoints dominated by Cuanzhu (BL2) for controlling intractable hiccups using a meta-analysis of RCTs.

\section{Methods}

\subsection{Design}

A systematic review and meta-analysis will be carried out in this study, which will only include RCTs and will exclude equivalence trials and clinical inferiority trials.

\subsection{Registration}

We have registered the protocol on the International Prospective Register of Systematic Reviews (PROSPERO) (registration number: CRD42020114900). This study will be conducted by strictly following the requirements of the Preferred Reporting Items for Systematic Reviews and Meta-Analyses (PRISMA) guidelines.[24] The summary of this study will follow the PRISMA for Protocol (PRISMA-P) guidelines.[25]

\subsection{Inclusion criteria}

This study strictly follows the following criteria for inclusion in the literature.

\subsubsection{Types of studies}

This study will be evaluated and screened according to the research review guidelines, including participants, interventions, comparisons, and outcomes.

Randomized controlled trials (RCTs) that have used acupuncture as a monotherapy or combined with other treatments to treat intractable hiccups will be included in the study. In case any RCT applied an incorrect random approach, then it will not be included. Furthermore, non-RCTs, animal experiments, human cell or tissue experiments, and repeated publication studies will be excluded.

\subsubsection{Types of participants}

Adult patients aged $>18$ years who meet the criteria for persistent and intractable hiccup diagnosis will be included, regardless of gender, nationality, and education level (refer to the Diagnostic Standard for the Clinical Diagnosis and Treatment of Digestive System Diseases[26] for intractable hiccups; the hiccups 
last for more than 1 month. They could have restarted after 30-60 min). Patients who do not meet the diagnostic criteria or have received other interventions will be excluded.

\subsubsection{Types of interventions}

Experimental group: This group will use acupuncture at acupoints dominated by Cuanzhu (BL2) to treat intractable hiccups based on the control group. The acupuncture method, treatment time, and treatment frequency are not limited.

Control group: This group will include a variety of different conventional treatments, such as no treatment, placebo, sham acupuncture, and drugs.

The routine treatment in the RCTs need not be necessarily similar, but the intervention method must be consistent.

Several comparisons will be analyzed as follows:

(1) Acupuncture at acupoints dominated by Cuanzhu (BL2) compared with no treatment

(2) Acupuncture at acupoints dominated by Cuanzhu (BL2) compared with placebo

(3) Acupuncture at acupoints dominated by Cuanzhu (BL2) compared with sham acupuncture

(4) Acupuncture at acupoints dominated by Cuanzhu (BL2) compared with drugs

\subsubsection{Types of outcome measures}

(1) Primary outcome measures

$\bigotimes$ The time when the hiccup symptoms disappear after interventions

$\bigotimes$ The frequency or some other change in hiccup symptoms after interventions

$\bigotimes$ The adverse events produced by the intervention

(2) Secondary outcome measures

QThe number of diverse methods of interventions that are needed to be taken to stop the hiccups

\Secondary adverse events that do not need withdrawal

\subsection{Information sources}

Eight electronic databases, including four Chinese databases (CNKI, SinoMed, Wanfang Database, and Chinese Scientific Journal Database (VIP)), four English databases (Web of Science, Medline, Embase, 
and Cochrane Library) will be searched from their date of establishment to September 2020. In addition, gray literature such as conference papers and bibliographic references will be included. The search was independently completed by two researchers in September 2020. Both Chinese and English RCTs will be included.

\subsection{Search strategies}

A comprehensive search strategy will be developed for PubMed or MEDLINE (Figure 2)

\subsection{Data collection and management}

\subsubsection{Selection of studies}

The evaluators are trained and prescreened to ensure the accuracy and standardization of the literature screening process. The literature screening process requires at least two reviewers ( $Z Y$, and WZJ) to conduct independent discussions and discuss the decision with a third commentator (QWX) whenever there is a disagreement. The literature screening process will be reported in detail in the systematic review plan and in the full text. The following steps are included (Figure 3):

(1) Using NoteExpress to classify and organize the initial inspection documents and eliminate duplicate documents

(2) Reading the title and abstract of each study and excluding unrelated studies that do not significantly meet the inclusion criteria

(3) Analyzing and removing repeated publications

(4) Trying to contact the original author to supplement relevant information for studies with incomplete information.

\subsubsection{Data extraction}

Two reviewers (ZY, and WZJ) will use the predesigned standardized information extraction form for extracting research data. The extracted data will include basic information (such as the title, published, journal, publication time, author, ID number, and author contact information, ), research methods (such as the inclusion or exclusion criteria, randomization methods, blinding, sample size, intervention group, and control group), intervention cycle, frequency of intervention, participant characteristics (such as age, gender, and grouping, ), and other confounding factors (such as the funding source, author's key conclusions, author's evaluation of confounding factors, and references to other studies, etc.).

\subsection{Assessment of risk of bias and reporting of study quality}

We intend to use the Risk of Bias Assessment tool recommended by the Cochrane Handbook 5.1 to evaluate the methodological quality of the included RCTs. 
The following seven aspects will be included:

(1) Random sequence generation (selection bias)

(2) Allocation concealment (selection bias)

(3) Blinding of participants and personnel (performance bias)

(4) Blinding of outcome assessment (detection bias)

(5) Incomplete outcome data (attrition bias)

(6) Selective reporting (reporting bias)

(7) Other bias

For each of the included studies, an evaluation of "yes" (low bias), "no" (high bias), and "unclear" (indeterminate lack of relevant information or bias) will be done according to the abovementioned seven terms. The decision will be discussed with the third commentator whenever there is a disagreement. The "Risks of bias graph" and "Risk of bias summary" tools in the RevMan software will be used to represent the percentage of each criterion for all the judged results ("Yes," "No," and "Unclear").

\subsection{Comprehensive statistical analysis of data}

Data analysis and meta-analysis will be performed using the RevMan 5.3 software through the Cochrane collaboration. We will determine the effect size on the basis of different data. The second classification results will be analyzed at a $95 \%$ confidence interval for relative risk (RR) and $95 \%$ confidence interval analysis for continuous variables using MD (Standard Deviation).

\subsubsection{Handling missing data}

If there are incomplete or missing data, we will try to contact the author by e-mail or phone to obtain the most complete data. In case complete data are not available, that particular RCT will be excluded.

\subsubsection{Heterogeneity analysis}

A chi-square test will be used to estimate the presence of statistical heterogeneity, and the $\mathrm{I}^{2}$ test will be used to estimate heterogeneity on R-3.3.2 software. If there is no heterogeneity, a fixed effects model $\left(I^{2}<50 \%, P>0.05\right)$ will be used. In case heterogeneity occurs, a random effects model will be used and a subgroup analysis or sensitivity analysis will be conducted.

\subsubsection{Subgroup analysis}

If the heterogeneity is obvious, a subgroup analysis will be performed based on different types of acupuncture (such as electroacupuncture, and fire needle, ) or other interventions using R-3.3.2 software. 


\subsubsection{Sensitivity analysis}

Sensitivity analysis will be performed to determine data reliability based on missing data, sample size, and heterogeneity using R-3.3.2 software.

\subsubsection{Publication bias}

A funnel plot will be used to determine if there is a publication bias when there are more than 10 studies included. In case of a publication bias, the funnel plot will appear as an asymmetric distribution.

\subsubsection{Grading of evidence quality}

The quality evaluation in this study will be completed using the Grading of Recommendations, Assessment, Development, and Evaluation established by the World Health Organization. The quality results will be divided into the following four aspects: "high," "medium," "low," and "very low."

\section{Discussion}

Persistent and intractable hiccups severely reduce the quality of life of patients. In recent years, a large number of studies on pharmacological and nonpharmacological treatments for this disease have been reported. However, there is a lack of a unified diagnostic method and treatment for intractable hiccups in the world. An increasing number of studies have reported that,[27] acupuncture can be used as a type of gastrointestinal response in the treatment of some pathological diseases to improve the quality of life of patients, such as intractable hiccups caused by stroke[20] or postoperative tumors.[28] Acupuncture applied at intractable hiccups points and the methods are diverse, and have obvious advantages of improving the curative effect and shortening the course of the disease.

Cuanzhu (BL2) is an empirical point for acupuncture treatment for intractable hiccups. According to the theory of Traditional Chinese Medicine, Cuanzhu (BL2) belongs to Bladder Meridian of Foot-Taiyang. The bladder meridian passes near the ridge which is connected with the diaphragm, spleen and stomach. Therefore, Cuanzhu (BL2) has the effect of anti-vomiting and stopping hiccups. It was reported that there were facial nerve and pulley nerve branches under Cuanzhu (BL2).[29] After stimulating local nerves, the high-level center of the cerebral cortex could be excited which would inhibit abnormal excitement of the vagus nerve, and alleviate diaphragmatic spasm.[26, 30] Cuanzhu (BL2) has been widely used in modern clinical treatment for various types of hiccups caused by encephalopathy, tumors, and other diseases. It is easy to operate and can be activated by pressing, which is suitable for adults and children. However, a systematic review and meta-analysis has not yet been conducted to evaluate the potential benefits and harms of applying acupuncture at acupoints dominated by Cuanzhu in the treatment of intractable hiccups. Therefore, this systematic review is aimed to provide a basis for clinicians to determine the benefits and harms of the treatment of hiccups and evidence for the clinical treatment of hiccups in the field of Chinese medicine, promoting the application of acupuncture at acupoints dominated by Cuanzhu to help more patients. 


\section{Ethics And Communication}

As this study does not involve raw data collection, no ethical review is required. The results of this study will provide strong evidence for the treatment of intractable hiccups using acupuncture applied at acupoints dominated by Cuanzhu. This will help clinicians to make treatment decisions for intractable hiccups. This systematic review will be published in a peer-reviewed journal and international academic conference.

\section{Strengths And Limitations}

This is the first systematic review and meta-analysis to synthesize the utility and safety of acupucturing acupoints dominated by Cuanzhu for improving intractable hiccups. This study can provide a basis for clinicians to determine the benefits and harms of the treatment of hiccups and evidence for the clinical treatment of hiccups in the field of Chinese medicine, improving the quality of life in patients. The methods and quality assessments follow the systematic review guidelines and criterion to minimize the risk of bias. The potential limitation of this study is that only studying the effect of acupoints dominated by Cuanzhu to treat intractable hiccups may cause certain language bias and different types of acupuncture may cause greater heterogeneity.

\section{Abbreviations}

RCT=randomized controlled trial, PRISMA-P=Preferred Reporting Items for Systematic Reviews and MetaAnalysis Protocol, $\mathrm{Cl}=$ confidence interval, GRADE=Grading of Recommendations, Assessment, Development, and Evaluation.

\section{Declarations}

\section{Author contributions}

Conceptualization: Weixun Qin, Yu Zhang.

Data extraction: Yu Zhang , Zhijie Wang.

Investigation: Mingming He, Xudong Jiang

Methodology: Zimeng Lv, Yu Zhang, Zhijie Wang

Project administration: Xudong Jiang

Resources: Mingming He, Qing Yuan

Software: Yu Zhang, Zimeng Lv, Qing Yuan

Writing-original draft: Gaiqin Yang, Yu Zhang, 
Writing-review \& editing: Weixun Qin, Gaiqin Yang

\section{Acknowledgements}

Not applicable.

\section{Funding}

Not applicable.

\section{Availability of data and materials}

Not applicable.

\section{Ethics approval and consent to participate}

Not applicable.

\section{Consent for publication}

Not applicable.

\section{Competing interests}

We have read and understood Medicine policy on the declaration of interests and declare that we have no competing interests.

\section{Data sharing}

No additional data are available.

\section{Open Access}

This is an Open Access article distributed in accordance with the terms of the Creative Commons Attribution (CC BY 4.0) license, which permits others to distribute, remix, adapt and build upon this work, for commercial use, provided the original work is properly cited. See: http://creativecommons.org/licenses/by/4.0/

\section{References}

1. Eroglu O. The effect of gender differences in protracted hiccups. Niger J Clin Pract 2018, 21(10):1356-1360.

2. Steger M, Schneemann M, Fox M. Systemic review: the pathogenesis and pharmacological treatment of hiccups. Aliment. Pharmacol. Ther. 2015, 42(9):1037-1050. 
3. Doshi $\mathrm{H}$, Vaidyalingam R, Buchan K. Atrial pacing wires: an uncommon cause of postoperative hiccups. Br J Hosp Med (Lond) 2008, 69(9):534.

4. Chou $\mathrm{CL}$, Chen $\mathrm{CA}$, Lin SH, Huang HH. Baclofen-induced neurotoxicity in chronic renal failure patients with intractable hiccups. South. Med. 2006, 99(11):1308-1309.

5. Chang FY, Lu CL. Hiccup: mystery, nature and treatment. J Neurogastroenterol Motil 2012, 18(2):123130.

6. Rey E, Elola-Olaso CM, Rodríguez-Artalejo F, Locke GR, 3rd, Díaz-Rubio M. Prevalence of atypical symptoms and their association with typical symptoms of gastroesophageal reflux in Spain. Eur $J$ Gastroenterol Hepatol 2006, 18(9):969-975.

7. Khorakiwala T, Arain R, Mulsow J, Walsh TN. Hiccups: an unrecognized symptom of esophageal cancer? Am. J. Gastroenterol 2008, 103(3):801.

8. Sampath V, Gowda MR, Vinay HR, Preethi S. Persistent hiccups (singultus) as the presenting symptom of lateral medullary syndrome. Indian J Psychol Med 2014, 36(3):341-343.

9. Cymet TC. Retrospective analysis of hiccups in patients at a community hospital from 1995-2000. J Natl Med Assoc 2002, 94(6):480-483.

10. Jeon YS, Kearney AM, Baker PG. Management of hiccups in palliative care patients. BMJ Support Palliat Care 2018, 8(1):1-6.

11. Kim JE, Lee MK, Lee DK, Choi SS, Park JS. Continuous cervical epidural block: Treatment for intractable hiccups. Medicine 2018, 97(6):e9444.

12. Kako J, Kobayashi M, Kanno Y, Tagami K. Intranasal Vinegar as an Effective Treatment for Persistent Hiccups in a Patient With Advanced Cancer Undergoing Palliative Care. J Pain Symptom Manage 2017, 54(2):e2-e4.

13. Zhang XP, Wang ML. Clinical observation on the treatment of hiccup after stroke by injection of metoclopramide combined with pressing Cuanzhu acupoints. Zhongguo Min Jian Liao Fa 2019, 27(07):24-26.

14. Li WH, Bai LJ. Acupuncture at Cuanzhu (B2) and Tianding (LI17) in the Treatment of Intractable Hiccup for 30 Cases. Zhongguo Zhong Yi Yao Xian Dai Yuan Cheng Jiao Yu 2018,16(10):128-129.

15. Wang B. 80 cases of intractable hiccup treated by acupuncture. Zhongguo Zhen Jiu 2011, 31(02):181-182.

16. Zhou XQ. Curative effect of electroacupuncture and ultrashort wave combined with intermediate frequency in the treatment of hiccup of 26 cases. Zhongguo She Qu Yi Shi 2017, 33(24):76-77.

17. Liu Y, Gao Y, Liu XJ. Ultrasound guided precise positioning and depth of electroacupuncture at $\mathrm{Ge}$ Shu point in treating intractable hiccup. Zhongguo Zhong Yi Yao Xian Dai Yuan Cheng Jiao Yu 2018, 16(10):120-122.

18. Jiang PJ, Deng W. Clinical observation on acupuncture combined with acupoint injection for treatment of 49 cases of intractable hiccup after stroke. An Mo Yu Kang Fu Yi Xue 2019, 10(05):1315. 
19. Deng HM, Tuerxun H. Long-term efficacy of embedding therapy for hiccup after stroke. Yunnan Zhong Yi Zhong Yao Za Zhi 2018, 39(09):63-64.

20. Yue J, Liu M, Li J, Wang Y, Hung ES, Tong X, Sun Z, Zhang Q, Golianu B: Acupuncture for the treatment of hiccups following stroke: a systematic review and meta-analysis. Acupunct Med 2017, 35(1):2-8.

21. Moretto EN, Wee B, Wiffen PJ, Murchison AG. Interventions for treating persistent and intractable hiccups in adults. Cochrane Database Syst Rev 2013, 2013(1):Cd008768.

22. Zhang ZY, Lian XL. A clinical analysis of treating 28 cases central intractable hiccups by press Cuanzhu point. Zhongguo Lin Chuan Yan Jiu 2014, 6(29):40-42.

23. Qiu XW, Hu QL, Yang XP. Clinical study on treatment of intractable hiccup after traumatic brain injury with special acupuncture. Shandong Yi Yao 2013, 53(47):38-39.

24. Moher D, Shamseer L, Clarke M, Ghersi D, Liberati A, Petticrew M, Shekelle P, Stewart LA. Preferred reporting items for systematic review and meta-analysis protocols (PRISMA-P) 2015 statement. Syst Rev 2015, 4(1):1.

25. Beller EM, Glasziou PP, Altman DG, Hopewell S, Bastian H, Chalmers I, Gøtzsche PC, Lasserson T, Tovey D. PRISMA for Abstracts: reporting systematic reviews in journal and conference abstracts. PLoS Med 2013, 10(4):e1001419.

26. Rouse S, Wodziak M. Intractable Hiccups. Current neurology and neuroscience reports 2018 , 18(8):51.

27. Ge AX, Ryan ME, Giaccone G, Hughes MS, Pavletic SZ. Acupuncture treatment for persistent hiccups in patients with cancer. J Altern Complement Med 2010, 16(7):811-816.

28. Kim TH, Kang JW, Lee MS. Current evidence of acupuncture for symptoms related to breast cancer survivors: A PRISMA-compliant systematic review of clinical studies in Korea. Medicine 2018, 97(32):e11793.

29. Liu YJ, Liu TJ. Study on the mechanism of stubborn hiccups treated by intensively pressing Zanzhu acupoints with two-step breathing regulation. Zhongguo Min Jian Liao Fa 2015, 23(02):42-43.

30. Gallagher J. Anterior and posterior diaphragm kinesio taping for intractable hiccups after ischemic stroke: A case report. Medicine 2018, 97(34):e11934.

\section{Figures}




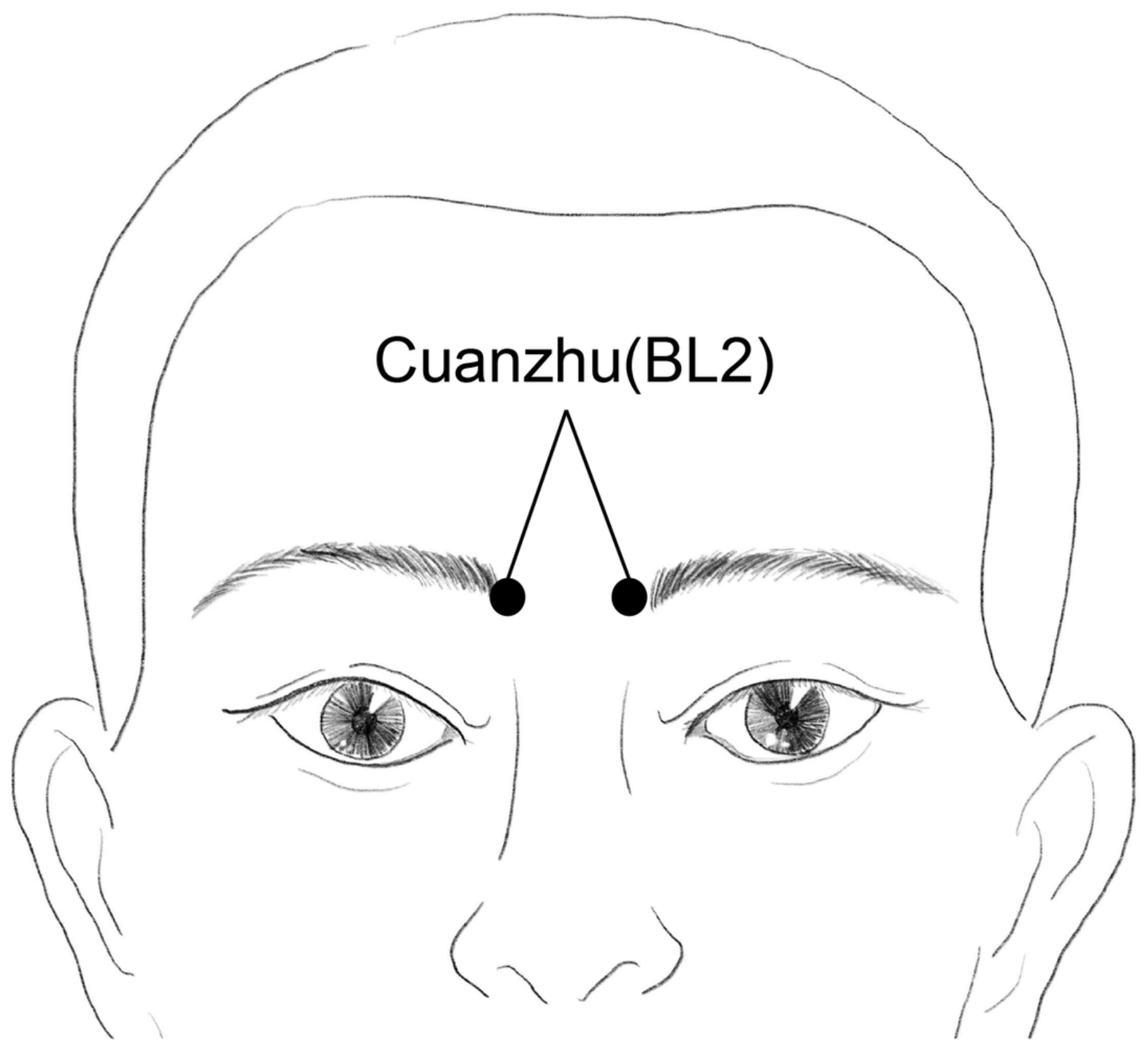

Figure 1

Location of Cuanzhu (BL2) 


\begin{tabular}{|c|c|c|c|}
\hline \multicolumn{2}{|c|}{$\begin{array}{l}\text { Search strategy and study } \\
\text { design filter for Medline which } \\
\text { was adapted for Embase, Web } \\
\text { of Science, Cochrane Library: }\end{array}$} & \multicolumn{2}{|c|}{$\begin{array}{l}\text { Cochrane highly sensitive } \\
\text { search strategy for identifying } \\
\text { randomized trials in MEDLINE: } \\
\text { sensitivity-maximizing } \\
\text { version(2008 revision); Ovid } \\
\text { format. } \\
\text { (Box 6.4.c of The Cochrane } \\
\text { Handbook of Systematic } \\
\text { Reviews of Interventions). }\end{array}$} \\
\hline $\mathrm{NO}$ & Search terms & NO & Search terms \\
\hline$\# 1$ & Hiccup/ & $\# 1$ & $\begin{array}{c}\text { randomized controlled } \\
\text { trial.pt. }\end{array}$ \\
\hline$\# 2$ & $\begin{array}{l}\text { (hic*up* or hic*ough* or } \\
\text { singult*).mp. }\end{array}$ & $\# 2$ & controlled clinical trial.pt. \\
\hline \#3 & $\# 1$ or \#2 & $\# 3$ & randomized.ab. \\
\hline$\# 4$ & Cuanzhu[All Fields] & $\# 4$ & placebo.ab. \\
\hline$\# 5$ & BL2[All Fields] & $\# 5$ & drug therapy.fs \\
\hline$\# 6$ & $\begin{array}{l}\text { point[All Fields] AND } \\
\text { BL2[All Fields] }\end{array}$ & \#6 & randomly.ab. \\
\hline$\# 7$ & $\begin{array}{l}\text { "Acupunctue } \\
\text { points"[MeSH Terms] OR } \\
\text { ("acupuncture"[All } \\
\text { Fields] AND “points"[All } \\
\text { Fields]) OR "acupuncture } \\
\text { points"[All Fields] OR } \\
\text { "acupoints"[All Fields] }\end{array}$ & $\# 7$ & trial.ab. \\
\hline \multirow[t]{4}{*}{$\# 8$} & \#3 AND \#7 & $\# 8$ & groups.ab. \\
\hline & & $\# 9$ & or/ $1-8$ \\
\hline & & $\# 10$ & $\begin{array}{l}\text { exp animals/ not } \\
\text { humans.sh. }\end{array}$ \\
\hline & & & 9 not 10 \\
\hline
\end{tabular}

\section{Figure 2}

Search strategies 


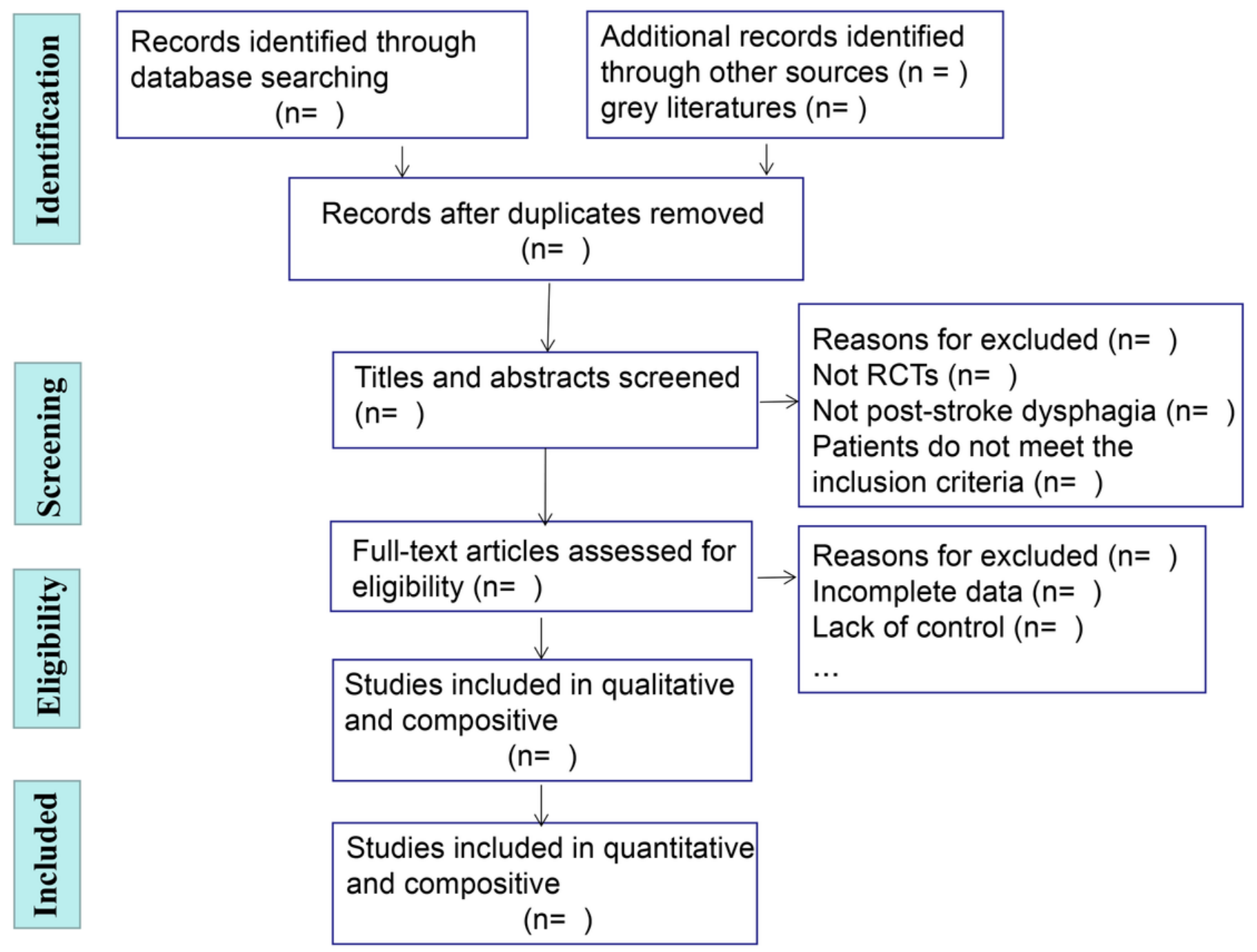

Figure 3

PRISMA flow chart of the study process

\section{Supplementary Files}

This is a list of supplementary files associated with this preprint. Click to download.

- PRISMAPchecklist.doc 